\title{
The Design of a High Performance MMW Radar System for Autonomous Land Vehicle Navigation
}

\author{
S.Clark and H.Durrant Whyte \\ Australian Center for Field Robotics, \\ Department of Mechanical and Mechatronic \\ Engineering, \\ University of Sydney, NSW 2006, Australia \\ E-mail: clark/hugh@mech.eng.usyd.edu.au
}

\begin{abstract}
This paper describes the design of a high performance $77 \mathrm{GHz}$ millimetre wave radar, signal processing and control system for use in autonomous vehicle navigation. The radar front end and intermediate frequency components are described together with a method of distinguishing pre-placed target beacons from other reflectors using the polarisation of the reflected signal. Digital signal processing hardware is described which allows reflected signal power to be determined at incremental distances from the radar. A control unit maintains a constant rotational velocity of a deflector plate positioned above the aperture and reads the azimuth of each radar sweep. In this way, range and bearing measurements are available to update a Kalman filter estimating vehicle location as it navigates around an environment.
\end{abstract}

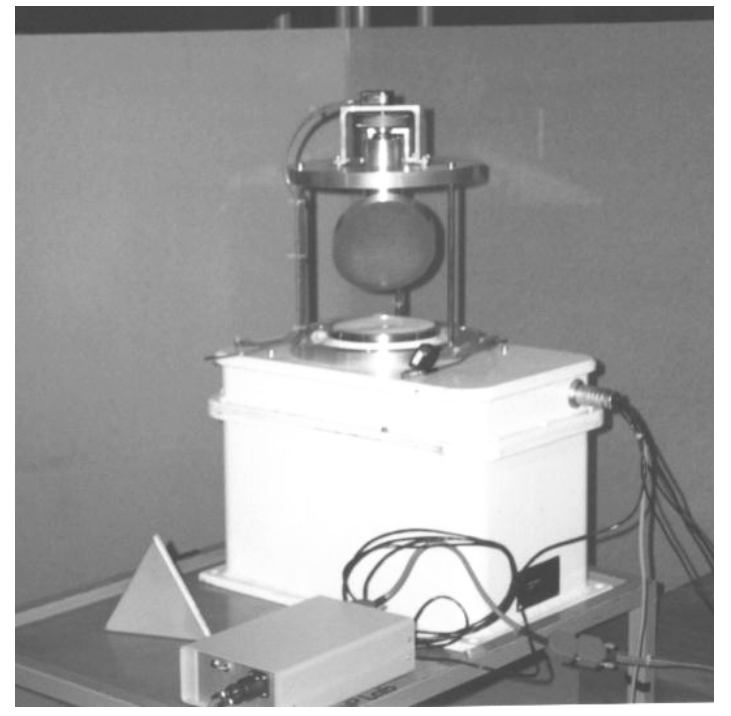

Fig. 1 Radar unit with rotating swash-plate beam deflector. The deflector introduces 6 degrees of vertical beam divergence.
This work was conducted as part of the "Field Automation" research program of the Cooperative Research Centre for Mining Technology and Equipment (CMTE)

\section{Introduction}

Millimeter wave (MMW) radar satisfies many of the criteria required of a sensor in high performance outdoor autonomous vehicle navigation. The short wavelength, compared to microwave radar provides high resolution measurements. MMW radar is also able to operate in adverse weather and environment conditions compared to optical sensors such as lasers. With modern digital processing techniques, it is possible to locate navigation targets at greater distances than other sensors with a high degree of accuracy. The potentially high bandwidth of the radar intermediate frequency (IF) allows measurement of sufficient range and resolution to maintain high quality, high rate updates for a vehicle navigation algorithm. Extra information can be extracted from the polarisation of the radar signal to enable navigation targets to be distinguished from clutter.

This paper describes a high-performance MMW navigation radar system exploiting many of the potential advantages of this type of radar as a primary navigation sensor. The system described differs substantially from the collision detection radars currently being developed for automotive applications in a number of ways. First, the unit has much higher range and bearing accuracies than those commonly deployed. Second, the system is capable both of high-precision position determination and of generating full amplitude radar images for use in terrain mapping.

The design and essential measurement principles of the front-end radar unit are described in Section II. The unit employs a saw-tooth FMCW measurement method with a swept bandwidth of $600 \mathrm{MHz}$, providing an unprocessed range accuracy of $0.25 \mathrm{~m}$. The front-end unit also exploits a dual polarisation receiver to discriminate between navigation targets and ground clutter. Section III describes the implementation of a signal processing system necessary to provide long range high accuracy navigation information and which meets the strict timing requirements necessary to maintain filter update frequency. Section IV presents the results of measurements made from both the position determination 
system and also the terrain images produced by the radar. In conclusion, Section $\mathrm{V}$ describes the applications for which this radar unit is intended.

$$
\begin{aligned}
& R=\frac{c T_{s}}{2} \frac{1}{f_{s}} \Delta f \\
& \Delta F=\frac{1}{T_{s}} \\
& \Delta R=\frac{c}{2 f_{s}}
\end{aligned}
$$

\section{Radar Design}

\subsection{Measurement Theory}

The radars employed in this paper has a $77 \mathrm{GHz}$ baseband frequency, the designated automotive frequency in Europe and the United States, chosen for its low atmospheric absorption [1]. Figure 2 shows how the carrier wave is frequency modulated (FMCW) over $600 \mathrm{MHz}$ in either $1 \mathrm{mS}$ or $500 \mathrm{uS}$ depending on which of two available prototype units are used. The reflected signal is also shown on an expanded time scale.

The time between transmission and reception of the radar signal gives a measure of the distance to the reflecting surface. However given the limited maximum range it is not practical to measure such small time intervals. Instead, transmitted and received MMW signals are mixed and the frequency difference extracted. This intermediate frequency is a measure of the target distance according to Equation 1, where $\Delta f$ is the intermediate frequency, $T_{s}$ the sweep time, $f_{s}$ swept frequency and $c$ the speed of electromagnetic radiation. From Equation 2, the smallest resolvable frequency for a $1 \mathrm{mS}$ sweep is $1 \mathrm{kHz}$ and thus the range resolution is obtained from Equation 3 as $0.25 \mathrm{~m}$. Note that the range resolution is dependent only on the swept bandwidth.

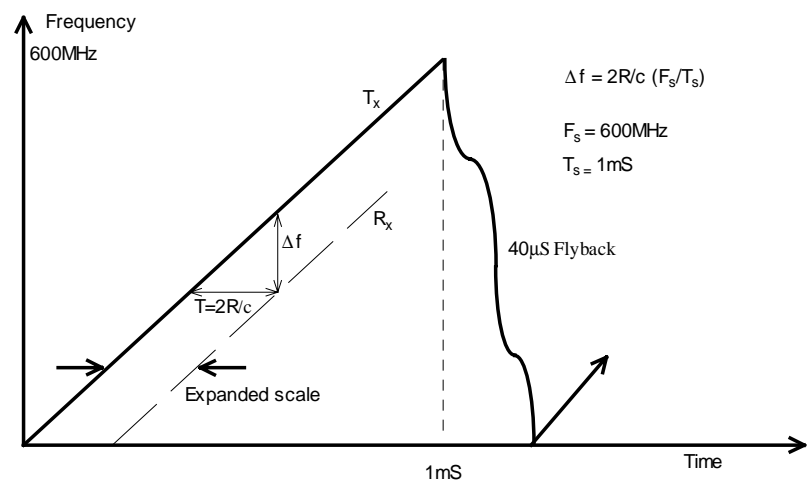

Fig. 2 Frequency difference gives range measurement.
Range resolution can be improved by curve fitting to the power spectral density data around the peak return, this is further discussed in section IV-D. The maximum range measurable depends on the maximum IF bandwidth that can be processed and the radar parameters $T_{s}$ and $f_{s}$.

The two MMW radar units described in this paper employ a $36 \mathrm{db}$ lens antenna yielding a beam divergence of approximately $1.2^{\circ}$. For this reason, a maximum radar range of 250 meters was selected since the beam width at this range will be in the order of meters making it difficult to accurately locate targets. For the $1 \mathrm{mS}$ sweep unit, a 250 meter range corresponds to a $1 \mathrm{Mhz}$ bandwidth. The same bandwidth with a $500 \mu \mathrm{S}$ sweeps gives a maximum range of 125 meters with the same 0.25 meter resolution. With this unit however the size of Fourier transform necessary will be halved, easing the required specification of the DSP and signal processing unit and enabling faster navigation filter update.

\subsection{Radio frequency components}

The radar returns are used either for location determination, by matching and triangulating pre-placed beacons to a map of their known co-ordinates, or to build a terrain map in front of the vehicle for either navigation or collision detection functions. To satisfy this dual purpose the radar was designed to enable location beacons to be distinguished from other terrain reflections. This is achieved using polarisation information.

The transmitter oscillator generates a vertically polarised signal, this passes through a circulator and is incident at $45^{\circ}$ to a quarter wavelength plate, a circularly polarised ${ }^{1}$ signal results, Figure 3 . The $\lambda / 4$ plate comprises a dielectric material that is adjusted to induce a $90^{\circ}$ phase shift in one of two orthogonal axis [7]. It is not necessary for the transmitted signal to be circularly polarised, only that 2 orthogonal signals of equal amplitude be produced. However a $\lambda / 4$ plate is a convenient way of achieving this. The target corner reflector incorporates a vertical polarising grid on the front face. The component parallel to the grid will be reflected and not enter the corner. Consequently the effective area of this component will be small compared to the orthogonal component which enters the corner. Furthermore the parallel component will not be reflected back to the radar unless the transmitted signal is normally incident, whereas the corner reflector returns the signal directly back along an incident path for a wide range of incident angles [8]

A signal reflected from a target will then have horizontal polarisation and be incident on the $\lambda / 4$ plate at $45^{\circ}$, again producing a circular polarisation in the received channel. This is directed to an orthomode transducer, which has the

1 A circular polarised signal consists of 2 orthogonal polarisation's of equal power with $90^{\circ}$ phase difference 
effect of separating horizontal and vertical components. Note, at this point only a signal that is purely horizontally (from a target) or vertically (from some other source) polarised will produce a circular polarisation out of the $\lambda / 4$ plate. Any other polarisation will make the output somewhat elliptical, and only the circular polarisation will be split into equal power in the horizontal and vertical channels by the orthomode transducer. Herein lies the means to distinguish navigation targets: Both channels are mixed to obtain the frequency difference and the power spectra produced. A comparison of the signal power levels enables navigation targets to be distinguished from other reflectors. Reflections that are deemed not to be from targets can be used to trigger collision avoidance procedures when they appear a short distance from the vehicle.

A single reflection from a flat surface at normal incidence will switch the polarisation of the incoming signal. Left circular polarised (LCP) transmitted signal will be directed back into the $\lambda / 4$ plate as right circular polarisation (RCP). The induced phase shift in one of the orthogonal components will produce a linear polarisation again and the orthomode transducer ensures this will appear in only one of the power spectra. The same situation occurs if the LCP transmitted signal undergoes an even number of reflections and returns LCP, the $\lambda / 4$ plate ensures that the orthogonal components are in phase or $180^{\circ}$ out of phase. In either case the result is a linear polarisation to the orthomode transducer and a power peak in a one channel only.

The occurrence of natural linear polarisers may be more common then expected as the signal wavelength is comparable to object dimensions in the environment. This methodology cannot be relied upon exclusively to determine polarised target from other reflections. A reflection from a hard vertical edge may be expected to increase the vertical component and diminish the horizontal. Should this occur to an extent that the reflection is almost entirely vertically polarised then the edge may be confused as a target.

\subsection{Intermediate frequency components}

The radar transmitter is based on a gallium arsenide Gunn diode oscillator that nominally delivers $10 \mathrm{~mW}$ of power. The mixer produces sum and difference components and a low pass filter removes all except for the intermediate frequency, the difference between transmitted and received signals as shown in Figure 4

Included in the radar IF signal path is an $\mathrm{R}^{4}$ filter, the transmitted signal spreads as $1 / R^{2}$ as does any reflected signal. Since range is proportional to frequency (Equation 1) the range dependence of the IF signal may be removed by an appropriate high-pass filter. The filter reduces the dynamic range necessary in the ADC required if power levels varied as $\mathrm{R}^{4}$. Finally the IF amplifier drives the signal processor.

\subsection{System Inaccuracies}

Linearity of the sweep is critical to ensure accurate range measurements, nominal linearity of $0.1 \%$ in the radar front end translates directly to the equivalent range accuracy. Range error introduced by the radar at 250 meters will nominally be $0.25 \mathrm{~m}$. The radar has been calibrated to check the deviation between measured and actual target separations.

An added factor to include in the target range determination is the Doppler frequency shift. When detecting targets only the vehicle will be moving but in the collision avoidance scenario the velocities of the vehicle and obstacle will determine the frequency shift. The latter is, of course, unlikely to be known accurately. Equation 4 gives the relationship between frequency shift and vehicle

Fig. 3 Polarisation for target discrimination

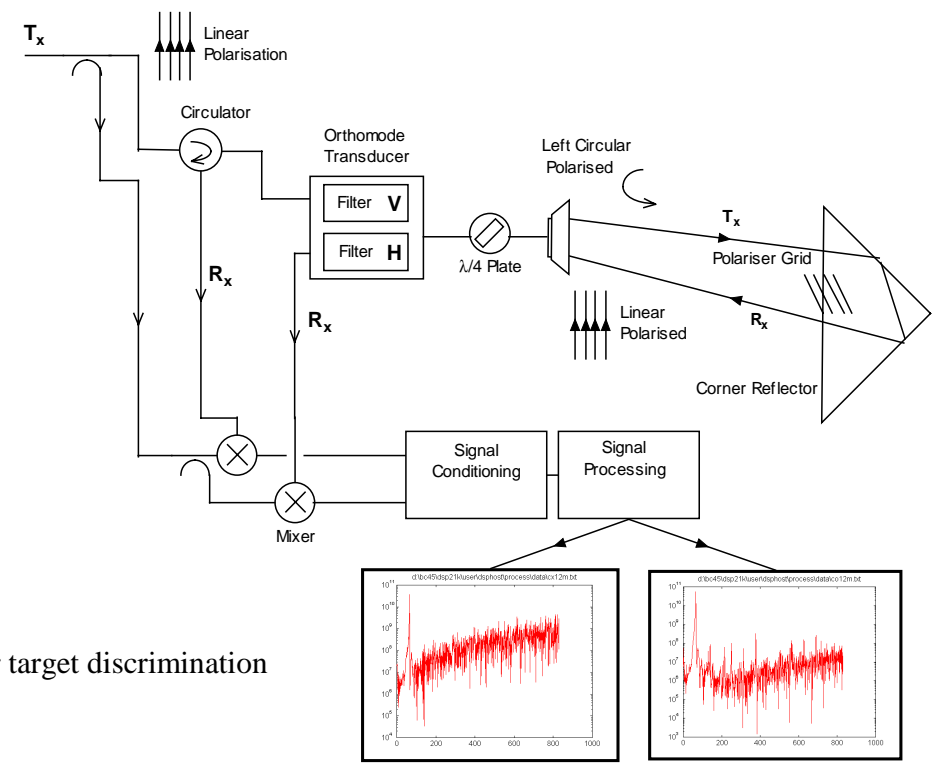




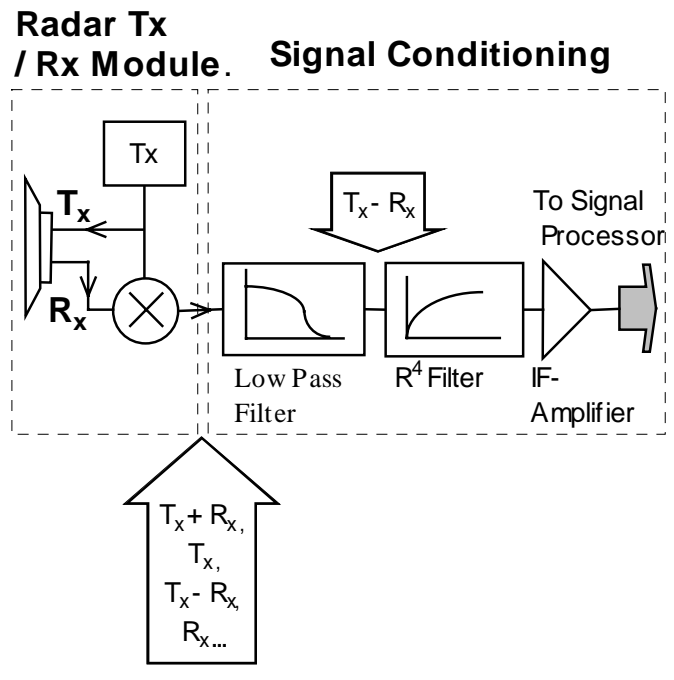

Fig. 4 Signal conditioning block diagram

velocity. For a vehicle directly approaching a stationary reflector at $20 \mathrm{kph}$ a frequency shift of $2800 \mathrm{~Hz}$ is induced, uncorrected this would give a 0.7 meter error in the range calculation.

$f_{\text {doppler }}=\frac{2 V}{\lambda}$

An alternative design of radar might employ a sweep up and down the frequency range. A Doppler shift in the received signal will cause an increase in one half of the cycle and a decrease in the other, target range can be extracted by averaging the two measurements $[1 \mathrm{pg} .83]$. Doppler shift can also be evaluated to give an indication of vehicle velocity at that instant. The downside of this approach is the extra processing involved measuring the two frequency sweeps. It is unlikely that time will be available to perform the extra Fourier transform and maintain the required update frequency of the navigation algorithm.

\section{Signal Processor System}

\subsection{Requirements of the signal processor}

Although the swept bandwidth is $600 \mathrm{MHz}$ the IF amplifier output rolls off by $3 \mathrm{~dB}$ at $2 \mathrm{Mhz}$. In the application described in this paper, it is unlikely that targets will be discernible above noise at ranges greater than $250 \mathrm{~m}$. Uncertainty as to target location because of the finite horizontal beamwidth gives little incentive to increase the IF bandwidth processed and hence the maximum range measurement. From Equation 1 then, it is necessary to measure frequencies up to $1 \mathrm{MHz}$. In addition to target location the radar data is intended for collision avoidance purposes so small area targets close to the unit should be discernible from the larger beacon signals without the latter saturating. An $\mathrm{AD}$ converter with 12bit dynamic range was chosen for this reason, collision avoidance with previous 8 bit designs had proved problematic.

The radar IF is sorted into frequency bins with an FFT algorithm to enable target range to be measured. This is performed on a high end DSP to meet the required specification of at least 250 processed measurements per second. Each channel of the radar IF is first filtered to remove aliases in the processing stage, the subsequent amplifier is adjusted to allow the full dynamic range of the ADC to be used. The DSP then must be capable of shifting data into the device, performing pre and post processing (including windowing and PSD calculations) and a $4 \mathrm{~K}$ FFT in $4 \mathrm{mS}$. The benchmark at $40 \mathrm{MHz}$ for a 4096 point real FFT is $1.81 \mathrm{mS}$, this allows ample time for pre and post signal processing. Should a further time reduction be necessary it is possible to process only part of the swept frequency, this effectively reduces the bandwidth and hence resolution but maintains the same maximum range measurement.

\subsection{Digital signal processor}

The DSP employed in the system is an Analog Devices 21062, the Super Harvard Architecture (SHARC), which lends itself to fast data transfer and processing. It is possible for example to move measurements from the ADC directly into memory (DMA) with no processor overhead, enabling the previous sample to be processed whilst the next is being set up. In the system described here, two 21062's are mounted on an ISA compatible board with hardware support to interface to a variety of I/O modules (in our case a two channel AD converter). Also included is a SHARCPAC interface allowing easy addition of up to four more processors into the system and $128 \mathrm{~K}$ of 48 bit wide, zero wait state SRAM is available on the carrier board.

In the application described here, one DSP is used for each radar channel and the results of each analysis are continually compared.

\subsection{Signal conditioning}

The ADC is clocked at $4.096 \mathrm{MHz}$ for the $1 \mathrm{mS}$ sweep radar, enabling sufficient samples to be collected for a $4 \mathrm{~K}$ FFT. The low pass filter has a cutoff frequency at $1 \mathrm{MHz}$ and a fast rolloff to $-72 \mathrm{~dB}$ by $3 \mathrm{MHz}$. Aliasing will occur from the Nyquist frequency $(2.048 \mathrm{MHz})$ and is attenuated to a negligible level by the $1 \mathrm{MHz}$ frequency bin, the 0 to $1 \mathrm{MHz}$ band will be without aliases. The filter output drives an amplification/buffer stage with selectable gain. To get full use of the 12bit ADC the amplifier must raise the radar signal close to the input voltage range of $2 \mathrm{Vpp}$ without saturating. It must also be designed for low noise, less than $72 \mathrm{~dB}$ for 12 bits over $2 \mathrm{~V}$. The prototype design did not quite achieve this, some noise being seen on the last two bits and future work may include an improved 
circuit design. The current design is more than adequate for system development but an improved amplifier may help for collision detection applications where it becomes necessary to discern targets of small radar cross-section (e.g. a man) against larger signals.

\subsection{Control unit}

A separate unit based on an embedded Z180 microcontroller was designed to control rotation of the deflector plate and fire the radar after programmed azimuth increments. All control and sequencing signals are sent to the radar and signal processor at RS422 levels. In addition a quadrature encoder attached to the rotating reflector plate allows azimuth for each radar sweep to be communicated to the DSP.

\subsection{Sequencing of signal processing}

To take advantage of the direct memory access (DMA) transfer capabilities of the SHARC processors two buffers are constructed in internal memory space. On the rising edge of a DVALID signal from the radar a DMA transfer is initiated from the ADC, this is completed after sufficient samples have been collected for a 4k FFT as shown in Figure 5. Signal processing starts at the end of the previous FFT/Host interrupt sequence. During this time an interrupt will be handled to upload the orientation of the reflector and then finally to initiate the next DMA transfer. In this way new data can be collected whilst the last readings are being processed.

The SHARC that handles the serial port (\#B) to read azimuth information is designated the slave and the other (\#A) which handles host communications, the master. Internal memory is available to each SHARC in multiprocessor memory space enabling the master to communicate azimuth and power in both channels to the host.

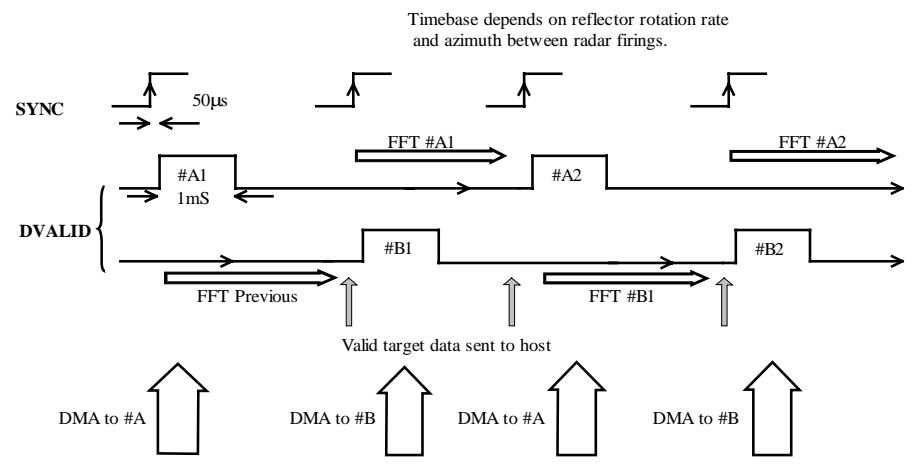

Fig. 5 DSP processing chain

\subsection{Signal processing program flow}

Once the power spectra of a collected chirp is calculated some target detection logic is applied. The processing on each SHARC is initiated by the same DVALID signal. When the slave of the two devices has finished a sample it sets a flag in the master's memory space if a potential target exists. It also sends the memory address of the PSD data, in this way the master SHARC can check for similar power levels in the same frequency bin to detect a beacon. If a reflection satisfies the beacon detection criteria then the host $\mathrm{PC}$ is interrupted with a range, bearing and a timestamp ${ }^{2}$ taken when the sample was collected. It is anticipated that a beacon will be detected at least 20 times a second and whilst for the purpose of system development this method is satisfactory. However, frequent interrupting of the host may slow the system by too much in time-critical applications. A future design may involve running the signal processor as an embedded system, eliminating overheads introduced by the host operating system.

\section{Results}

\subsection{Target design}

A trihedral corner reflector was used for testing the system. Large effective areas can be achieved for small physical trihedral sizes. Further, radiation incident on a trihedral is returned directly back along the incident path. The corner reflector has a $3 \mathrm{~dB}$ beamwidth ${ }^{3}$ of about 45 degrees in horizontal and vertical axis [8], consequently there are no special alignment problems between radar and reflector.

Several designs were considered for an omnidirectional reflector, these included a collection of corner reflectors and a diablo design. Finally, a design incorporating a diplane with one curved face was chosen. Four of these arranged in a cross provide a sufficiently large effective area from most orientations in the same plane. The curved faces provide some beamwidth in the axis perpendicular to the seam, unlike a pure diplane that has very narrow beamwidth in this axis. [8]. At the slight expense of physical size compared to a corner reflector an omnidirectional target beacon can be easily constructed.

\subsection{Stationary measurements}

A sequence of measurements were taken in the field whilst the deflector plate, shown in Figure 1, was stationary. Figure 6 shows measurements made of a $250 \mathrm{~m}^{2}$ target at

\footnotetext{
${ }^{2}$ The timestamp is generated by the ADSP on-chip reload timer

${ }^{3}$ Beamwidth refers to the responsive angle of the reflector.
} 
$250 \mathrm{~m}$ and $120 \mathrm{~m}$ from the radar. The abscissa is scaled in frequency bins and since the clocking frequency is 4.096 $\mathrm{MHz}$ in all these examples each bin represents $1 \mathrm{kHz}$ or $0.25 \mathrm{~m}$. The ordinate gives signal power. A major peak appears in the bin corresponding to the target location at 1000 and 480 respectively and is of similar power in both channels. The peak power in channel \#1 (co-planar) is over $20 \mathrm{~dB}$ above the noise floor at this range making it easily discernible from clutter. Signal to noise ratio is lower in channel\#0 (cx-planar), this is believed to be due to degradation of the mixer diodes in the front end and will be fixed in future units.

A series of range calibration tests were conducted inside a warehouse. The target was always clear in the signal but the corner of the building also presented a large cross section. An internal corner provides a large radar target and in some of the tests the signal exceeded the maximum range of the ADC. This can be problematic as harmonics of reduced amplitude appear around the peak and may be confused with target signals. Care has to taken to prevent signal saturation.

\subsection{Polarising grid effectiveness}

As discussed in section II-B the purpose of the target polarising grid is to produce equal power in both cross and co planar reflection peaks. The measured signal powers for the $250 \mathrm{~m}^{2}$ target at various ranges are given in the table 1 . Generally the power in the Co-planar channel is 3.7 times that in the cx-planar, this may be due to differing gains and component tolerances in the signal paths of each channel. Peak power was measured in co and cx channels for a $250 \mathrm{~m}^{2}$ target in the lab, the ratio of peak powers Co was 7.7. When the grid is removed the cx channel has maximum power and the ratio becomes 0.42 . Clearly the polarising grid influences relative power levels, with $12.5 \mathrm{~dB}$ difference between the 2 cases. The problem becomes one of establishing a reliable correction factor to give equal power in the polarised case. Once this is done, comparison of the power levels may successfully be used to identify targets from clutter. This will greatly reduce the number of false targets identified that must then

\begin{tabular}{|l|l|l|l|}
\hline $\begin{array}{l}\text { Distance } \\
(\mathrm{m})\end{array}$ & $\begin{array}{l}\text { Cx power } \\
\times 10^{11}\end{array}$ & $\begin{array}{l}\text { Co power } \\
\times 10^{11}\end{array}$ & Ratio \\
\hline 75 & 1.15 & 5.14 & 4.47 \\
\hline 60 & 4.1 & 24 & 5.85 \\
\hline 55 & 1.86 & 6.0 & 3.23 \\
\hline 45 & 1.2 & 3.4 & 2.62 \\
\hline 30 & 2.0 & 6.8 & 3.40 \\
\hline 15 & 0.71 & 1.76 & 2.46 \\
\hline
\end{tabular}

Table 1 Power levels in both channels at various ranges be discarded by the position determination filter.

\subsection{Target detection}

The bin width for the Fourier transform processing is $1 \mathrm{kHz}$, corresponding to $0.25 \mathrm{~m}$. The FFT algorithm gives a maximum in a particular bin for frequencies half a bin width above and below. $19.6 \mathrm{kHz}$ for example gives a maxima in the $20 \mathrm{kHz}$ bin as does $20.3 \mathrm{kHz} .19 .5 \mathrm{kHz}$ gives equal maximas in the 19 and $20 \mathrm{kHz}$ bins, although the peak power is less far away from a bin boundary. For frequencies that fit into the sample interval in an integer
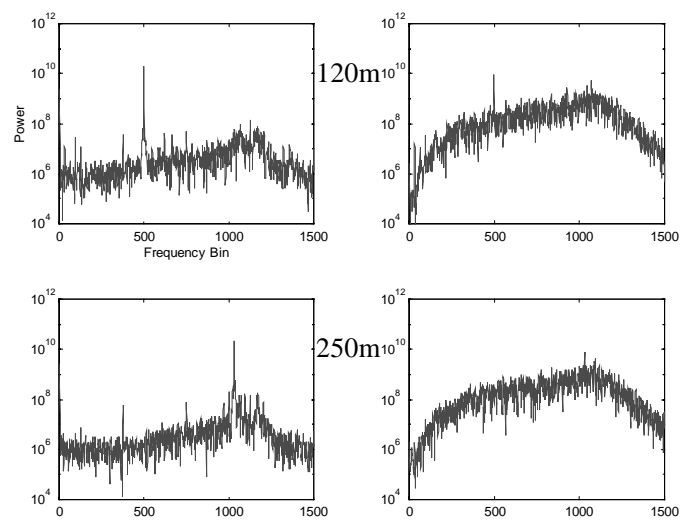

Fig. 6 PSD's in co and cx channels from targets at 120 and 250 meters

number of cycles the transform is well behaved, with a peak power in the bin representing that frequency and no signal leakage. When a non-integer number of cycles occur in the sample interval leakage occurs and the peak spreads into adjacent bins. To minimise this effect a windowing function can be used to reduce the influence of samples taken at the start and end of the window. Different functions have different properties, in these examples the Blackman window was chosen

As mentioned the peak power changes dependent on the proximity of the frequency to a bin boundary. Since cx and co planar reflections occur at the same frequency the ratio of powers remains constant.

Using peak power to determine distance restricts range accuracy to $0.25 \mathrm{~m}$. This can be improved by curve fitting to values either side of the peak. Whilst it is important to determine range with a high degree of accuracy it is also important to maintain the navigation algorithm update rate. It is unlikely that there will be time to fit high degree polynomials to the radar peaks in real time. The improvement in range resolution by fitting a quadratic to the data [11 Page 1002] was investigated with a range of simulated frequencies. Peak power gives range to \pm half a bin, the quadratic gives range to \pm 0.136 of a bin or within $68 \mathrm{~mm}$. 
Various methods have been investigated to detect targets from background clutter. The least processor intensive is simple threshold detection, a suitable level can be determined from examining the static power spectra for the calibrated reflector. Comparison of power levels as previously discussed will further reduce the possibility of false target detection. Strict acceptance tests in the Kalman filter updating the vehicle position reduces to a negligible level the possibility of a false target assignment. Another detection methodology is based on the well know radar technique of constant false alarm rate (CFAR) [8]. A cell can be considered as the area covered by the beamwidth over a single bin. A threshold for the cell under test is found using the mean and variance of the signal in adjacent cells. In this way clutter is quantitatively evaluated and a lower SNR is required to detect a target. This method requires more memory and processor cycles and overall was found less reliable than simple threshold detection. It may show superior performance during field trials and the possibility of using CFAR has not been completely discarded.

\subsection{Calibration}

Physical range as measured with a tape was compared with range from the radar for distances from 15 to 75 meters, curve fitting was used to refine distances between bins. A least squares analysis of this data gives an offset of 0.05 and a gradient of 0.97 , this is close to the expected value. The resolved frequency measurement and the physical distance are linearly related, making calibration a simple matter.

The effectiveness of the curve fitting algorithm is tested together with the linearity of the sweep by moving the target in $0.10 \mathrm{~m}$ increments from 45 to 46 meters [table 2] Generally the radar calculated and physically measured

\begin{tabular}{|l|l|l|l|}
\hline $\begin{array}{l}\text { Actual } \\
\text { Distance } \\
(\mathrm{m})\end{array}$ & $\begin{array}{l}\text { Peak } \\
\text { power } \\
(\text { Bin })\end{array}$ & $\begin{array}{l}\text { Quadratic } \\
\text { fitted (Bin) }\end{array}$ & $\begin{array}{l}\Delta \text { Distance } \\
(\mathrm{m})\end{array}$ \\
\hline 45.0 & 186 & 185.54 & 0.11 \\
\hline 45.1 & 186 & 185.98 & 0.10 \\
\hline 45.2 & 186 & 186.38 & 0.05 \\
\hline 45.3 & 187 & 186.58 & 0.11 \\
\hline 45.4 & 187 & 187.02 & 0.13 \\
\hline 45.5 & 188 & 187.53 & 0.10 \\
\hline 45.6 & 188 & 187.95 & 0.11 \\
\hline 45.7 & 188 & 188.40 & 0.14 \\
\hline 45.8 & 189 & 188.95 & 0.03 \\
\hline 45.9 & 189 & 189.05 & 0.10 \\
\hline 46.0 & 189 & 189.45 & \\
\hline
\end{tabular}

Table 2 Measured range resolution. distances match well, the overall distance between the 45 and 46 meter markers is $0.98 \mathrm{~m}$ as measured by the radar.

\subsection{Image data}

It has been possible to store successive power spectra in the DSP board SRAM. After the radar has scanned a segment of interest, data can be downloaded to the host computer to display. The plot in Figure 7 was generated in front of a stack of shipping containers. The lighter shading indicates higher amplitude in the power spectra. This corresponds to the edges of the containers. Notice at $25 \mathrm{~m}$ and $30^{\circ}$ no reflection is observed as there is no container at this point. In addition, behind this gap the signal was reflected from a number of other surfaces, whilst the adjacent segments show few reflections after the first surface. The container at $35 \mathrm{~m}, 30^{\circ}$ was only stacked one high and part of the radar signal had diverged sufficiently at this range to be reflected from surfaces beyond the container. The first containers in the segments at $15^{\circ}$ and $45^{\circ}$ were stacked three high.

\subsection{Sources of error}

Discrepancies between physically measured and radar measured distances may occur for several reasons. If the swept frequency is not exactly $600 \mathrm{MHz}$ an offset will be introduced to the range calculation, Equation 1. Also the distance between the radar transmitter and the swash plate including the wave-guides is not included in the distance calculation and will introduce an offset. Any departure from linearity in the sweep generator will produce small frequency shifts in the power spectrum and hence decrease range accuracy. Possible multiple paths must be considered when interpreting power spectra, the transmitted or received signal may bounce off walls or even the ground. The path between target and reflector will not then be the most direct, giving inaccurate range measurements. Fortunately the power shown for such paths is less than the power from the direct path. When the radar is close to a reflector, less than 20 meters for example, peaks have been seen in the power spectra at multiples of the initial larger peak. These are probably caused be caused by multiple transitions between deflector plate and target, diminishing in amplitude each time.

\section{Conclusions}

A signal processor has been designed and tested that will enable the IF of a millimetre wave radar to be processed. The dynamic range and fast operation of the system will enable a $77 \mathrm{GHz}$ radar to be used for the purposes of both navigation and collision detection. Measurements have been taken verifying the performance of the equipment and range data has been calibrated against physically measured ranges. Curve fitting methods have been 
investigated enabling range to be measured within $0.06 \mathrm{~m}$ over $250 \mathrm{~m}$

Enough is known about the expected power levels from calibrated targets to implement the target detection philosophy in the case of a rotating deflector plate. The unit will be used in future work both to provide position location information and terrain data for a number of autonomous outdoor vehicle projects.

\section{REFERENCES}

[1]M.I.Skolnik, "Introduction to Radar Systems Second edition," McGrawHil,1 1980

[2]H. Durrant-Whyte, "Proposal for the development of a $77 \mathrm{GHz}$ Radar Positioning System," Firefly Ltd., November 121992

[3]H.Durrant-Whyte, "An Autonomous guided vehicle for cargo handling applications,", The International Journal of Robotics Research, vol 15, No. 5, pp 407-440, October 1996

[4]R.E.Collin, "Foundations for microwave engineering," McGraw-Hill International Editions, second edition, 1992
[5]R.G.Carter, "Electromagnetic waves," Chapman and hall, 1990

[6]A.M.Bøifot, E.Lier, T.Schaug-Pettersen, "Simple and Broadband Orthomode transducer," IEE Proceedings, volume 137, Pt. H, No. 6, December 1990

[7]C.G.Montgommery, "Principles of microwave circuits," Radiation Laboratory series, McGraw Hill, 1948

[8]C.Currie, "Principles and applications of mmWave radar," Artech House, 1987

[9]G.Linfield, J.Penny, "Numerical methods using MATLAB," Ellis Horwood, 1995

[10]R E Ziemer, W H Tranter and D R Fannin, "Signals and Systems continuos and discrete," Macmillan, 1993

[11]E.Kreyszig, "Advanced Engineering Mathematics seventh edition," Wiley, 1993

[12]D.Langer, "An integrated MMW radar system for outdoor navigation," proceedings of the 1996 IEEE International conference on robotics and automation, Minneapolis, Minnesota, April 1996

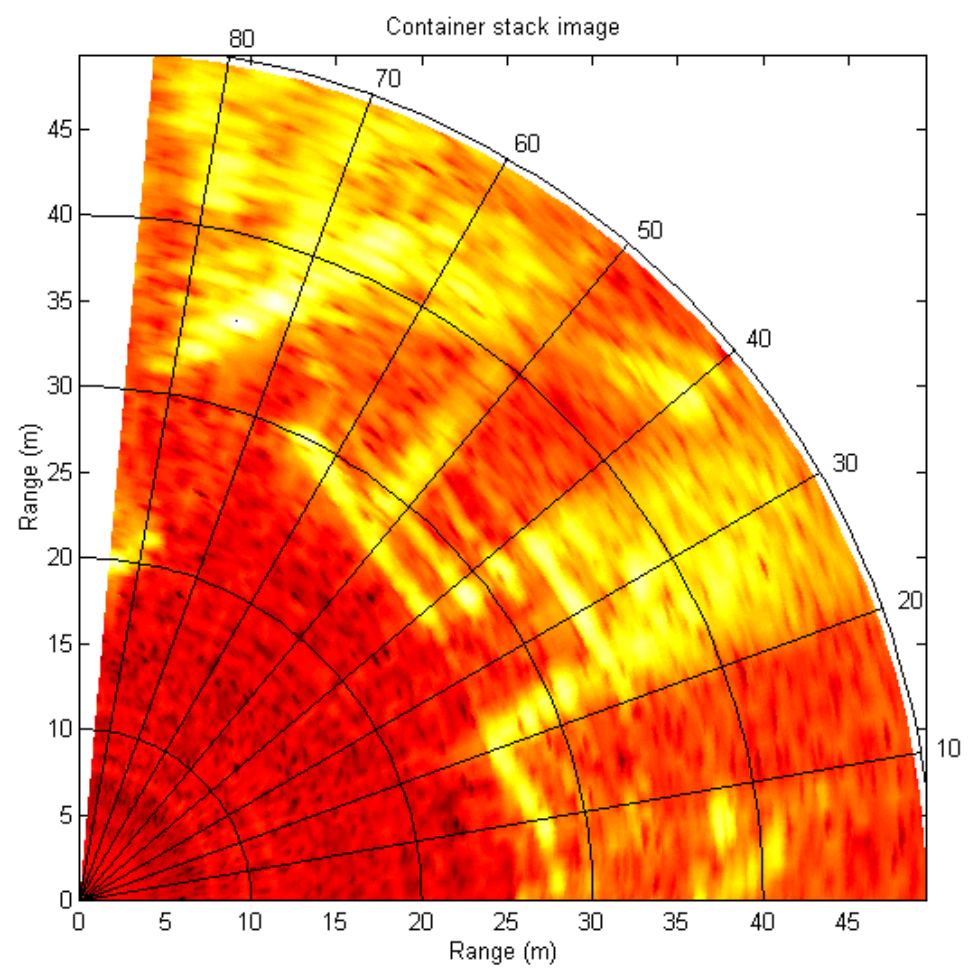

Fig. 7 Imaged container stack from successive power spectra 